2018

\title{
Single missing data imputation in PLS-based structural equation modeling
}

Ned Kock

Texas A \& M International University, Laredo, nedkock@tamiu.edu

Follow this and additional works at: https://digitalcommons.wayne.edu/jmasm

Part of the Applied Statistics Commons, Social and Behavioral Sciences Commons, and the Statistical Theory Commons

\section{Recommended Citation}

Kock, N. (2018). Single Missing Data Imputation in PLS-based Structural Equation Modeling. Journal of Modern Applied Statistical Methods, 17(1), eP2712. doi: 10.22237/jmasm/1525133160

This Invited Article is brought to you for free and open access by the Open Access Journals at DigitalCommons@WayneState. It has been accepted for inclusion in Journal of Modern Applied Statistical Methods by an authorized editor of DigitalCommons@WayneState. 


\section{Single missing data imputation in PLS-based structural equation modeling}

\section{Cover Page Footnote}

The author is the developer of the software WarpPLS, which has over 7,000 users in more than 33 different countries at the time of this writing, and moderator of the PLS-SEM e-mail distribution list. He is grateful to those users, and to the members of the PLS-SEM e-mail distribution list, for questions, comments, and discussions on topics related to SEM and to the use of WarpPLS. 


\title{
INVITED ARTICLE Single Missing Data Imputation in PLS- based Structural Equation Modeling
}

\author{
Ned Kock \\ Texas A\&M International University \\ Laredo, TX
}

\begin{abstract}
Missing data, a source of bias in structural equation modeling (SEM) employing the partial least squares method (PLS), are commonly handled with deletion methods such as listwise and pairwise deletion. Missing data imputation methods do not resort to deletion. Five single missing data imputation methods are considered employing the PLS Mode A algorithm of which two hierarchical methods are new. The results of a Monte Carlo experiment suggest that Multiple Regression Imputation yielded the least biased mean path coefficient estimates, followed by Arithmetic Mean Imputation. With respect to mean loading estimates, Arithmetic Mean Imputation yielded the least biased results, followed by Stochastic Hierarchical Regression Imputation and Hierarchical Regression Imputation. Single missing data imputation methods perform better with PLS-SEM based on their performance with other multivariate analysis techniques such as multiple regression and covariance-based SEM.
\end{abstract}

Keywords: Partial least squares; structural equation modeling; missing data imputation; path bias; stochastic regression; Monte Carlo simulation

\section{Introduction}

The method of partial least squares (PLS) experienced explosive growth in the context of structural equation modeling (SEM), whereby latent variables are measured via indicators in questionnaires (Akter et al., 2017; Kock, 2016; Rigdon, 2016). Indicators frequently take the form of scores generated based on questionstatements answered on Likert-type scales. PLS-SEM estimates latent variables

doi: 10.22237/jmasm/1525133160 | Accepted: December 5, 2017; Published: June 7, 2018.

Correspondence: Ned Kock, nedkock@tamiu.edu

Ned Kock is Killam Distinguished Professor and Chair of the Division of International Business and Technology Studies, A.R. Sanchez, Jr. School of Business, Texas A\&M International University. 


\section{NED KOCK}

through composites, which are exact linear combinations of the indicators assigned to the latent variables (Kock, 2015a; 2015b).

A main source of bias in PLS-SEM is missing data (Newman, 2014). Among patterns of missing data, particularly common in behavioral research is that known as missing at random (MAR), which is actually a misnomer. This pattern occurs when the probability of a missing value is related to other measured variables, but unrelated to the underlying values of the variable that are missing. For example, if scores measuring the accuracy of a graphical representation are more likely to be missing for a certain type of representation than for others, then the corresponding missing data will follow the MAR pattern. Researchers have traditionally used deletion methods, often listwise and pairwise deletion (Enders, 2010). They are a source of error that may distort coefficients of association; where the error is introduced into the data as deletion occurs. For example, missing data may be associated with groups of respondents who share some characteristics, and whose exclusion from datasets can significantly influence the strength of relationships among variables. Deletion methods also reduce the sample size available for an analysis, and thus the statistical power of virtually any type of analysis applied to the data. Wilkinson (1999) opine these techniques are "among the worst methods available for practical applications" (p. 598).

Missing data imputation methods provide an alternative to deletion methods. Through imputation missing data elements are replaced with well-informed guesses, obtained through various algorithms, leading to no reduction in sample size. Five single missing data imputation methods are considered in the context of PLS-SEM, with MAR data, of which two are new.

\section{Illustrative Model}

An illustrative model serves as the basis for a Monte Carlo experiment and empirical illustration. The illustrative model is depicted in Figure 1, and contains five latent variables, for which composites are estimated via PLS-SEM. The latent variables, which refer to theoretical constructs, are: communication flow orientation $\left(C_{1}\right)$, usefulness in the development of information technology (IT) solutions $\left(C_{2}\right)$, ease of understanding $\left(C_{3}\right)$, accuracy $\left(C_{4}\right)$, and impact on redesign success $\left(C_{5}\right)$. 


\section{SINGLE MISSING DATA IMPUTATION IN PLS-BASED SEM}

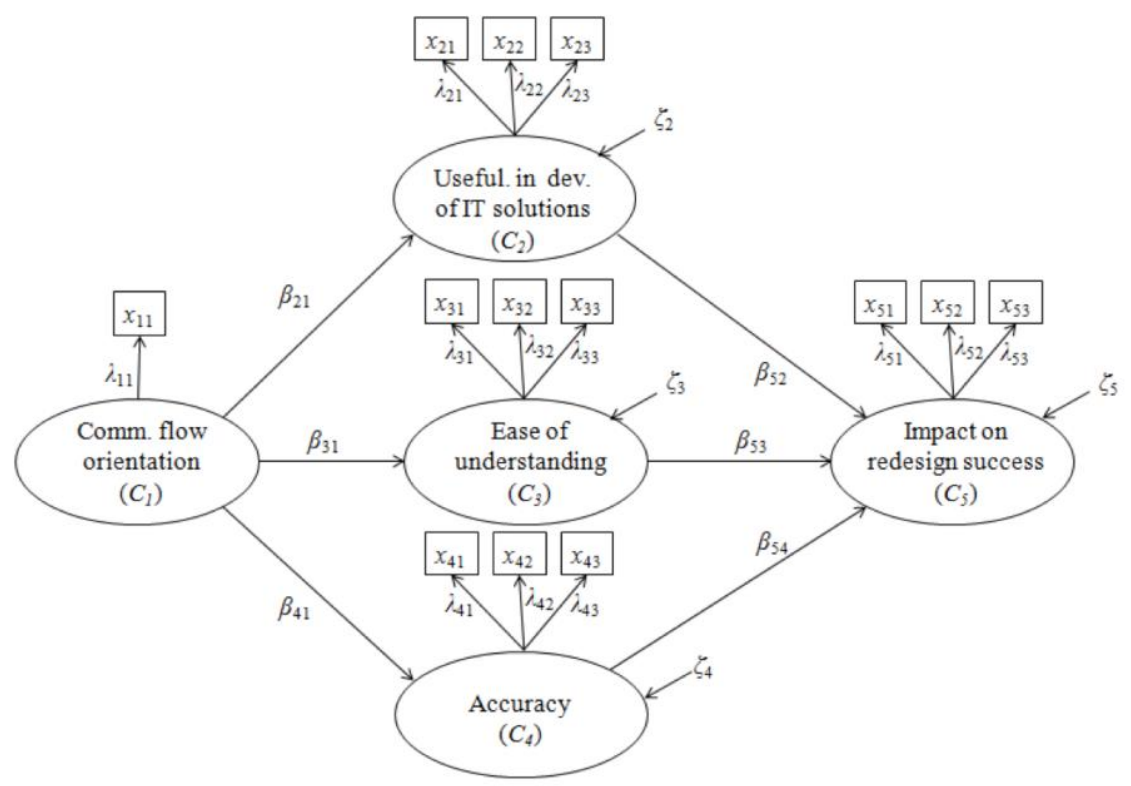

Figure 1. Illustrative model

The mathematical symbols used in the model, and in the following sections, were adapted from the classic path analysis, covariance-based SEM, and PLS literatures (Kline, 2010; Kock, 2016; Lohmöller, 1989; Wright, 1934; 1960): $\beta_{i j}$ is the path coefficient for the link going from composite $C_{j}$ to composite $C_{i}, \lambda_{i j}$ is the loading for the $j^{\text {th }}$ indicator of composite $C_{i}$, and $\zeta_{i}$ is the structural error associated with an endogenous composite $C i$. With exception of communication flow orientation $\left(C_{1}\right)$, a set of indicators $x_{i j}$ is used to measure each composite $C_{i}$. When more than one indicator is used to measure a composite, each indicator is assumed to measure the composite with a certain degree of imprecision.

Communication flow optimization theory (Danesh-Pajou, 2005; Kock, 2003) is the foundation on which the illustrative model is built. Although this theory is not the focus of the investigation, it is useful to know its main prediction. A greater focus on how communication takes place in business processes, in redesign efforts, is associated with better business process redesign outcomes. Business process redesign efforts are aimed at improving the operations of organizations, regardless of size and industry. In them groups of employees and managers collaboratively analyze and redesign business processes, which are sets of interrelated activities (Kock, 2007; Mendling et al., 2012). Virtually any good 


\section{NED KOCK}

or service is produced in organizations via a business process - e.g., the process of assembling a car, carried out by an automaker.

Communication flow orientation $\left(C_{1}\right)$ is the degree to which a business process modeling approach explicitly shows how communication interactions take place in a business process. This latent variable can be measured through a single indicator storing either 1 or 0 , for a study contrasting two opposite modeling approaches, corresponding to a high or low communication flow orientation of a business process modeling approach used.

Usefulness in the development of IT solutions $\left(C_{2}\right)$ is the degree to which a process modeling approach is useful in the development of a generic IT solution to automate the redesigned process. The need to automate redesigned processes with IT is almost universal in modern businesses. An example of questionstatement that can be used for measurement of this latent variable is: "This process modeling approach is useful in the development of a generic IT solution to automate the redesigned process".

Ease of understanding $\left(C_{3}\right)$ is the degree to which a process modeling approach is perceived to yield a process representation that is easy to understand. An example of question-statement that can be used for measurement of this latent variable is: Processes modeled using this approach are easy to understand.

Accuracy $\left(C_{4}\right)$ is the degree to which a process modeling approach is perceived to lead to an accurate representation of the process. An example of question-statement that can be used for measurement of this latent variable is: This process modeling approach leads to accurate process representations.

Impact on redesign success $\left(C_{5}\right)$ is the degree to which the process modeling technique used is perceived to lead to an actual improvement of the targeted business process. An example of question-statement that can be used for measurement of this latent variable is: Using this process modeling approach is likely to contribute to the success of a process redesign project.

\section{Missing Data Imputation Methods Analyzed}

All variables are assumed to be standardized. This has no effect on the implementation of the methods; the methods can take as inputs unstandardized variables, store means and standard deviations for later unstandardization, standardize the variables, apply the various operations that define the methods, and finally unstandardize the variables again prior to generating the outputs. 


\section{SINGLE MISSING DATA IMPUTATION IN PLS-BASED SEM}

\section{Arithmetic Mean Imputation}

Let xi be a column vector denoting one of the $\mathrm{k}$ manifest variables used in a SEM model. The Arithmetic Mean Imputation (MEAN) method assigns values to each missing element $\dot{x}_{i r}$ according to (1), where $N_{m}$ is the number of missing values in $x_{i}$, and $\bar{x}_{i}$ is the arithmetic mean of variable $x_{i}$.

$$
\begin{gathered}
\dot{x}_{i r}=\bar{x}_{i}, \\
r=1 \ldots N_{m} .
\end{gathered}
$$

The Arithmetic Mean Imputation (MEAN) method replaces each missing element $\dot{x}_{i r}$ in a column of data $i$ within a dataset, which refers to a manifest variable, with the average (or arithmetic mean) of that column. This method is the simplest of the imputation methods discussed here. Although it can be employed by itself, this method also plays an ancillary role in other methods.

\section{Multiple Regression Imputation}

The Multiple Regression Imputation (MREGR) method assigns values to each missing element $\dot{x}_{i r}$ according to (2), where $k$ is the number of manifest variables used in a model, $N_{m}$ is the number of missing values in $x_{i}$, and each of the elements of the matrix of estimated regression coefficients $\hat{\beta}_{x_{i} x_{j}}$ is calculated through a multiple regression analysis with $x_{i}$ as the criterion and $x_{j}(j=1 \ldots k, j \neq i)$ as the predictors.

$$
\begin{gathered}
\dot{x}_{i r}=\sum_{j=1}^{k} \hat{\beta}_{x_{i} x_{j}} x_{j r}, \\
j=1 \ldots k, j \neq i, r=1 \ldots N_{m} .
\end{gathered}
$$

In the Multiple Regression Imputation (MREGR) method each missing element $\dot{x}_{i r}$ is replaced with the corresponding expected value of $x_{i}$ given all of the other variables $x_{j}(j=1 \ldots k, j \neq i)$ in the dataset. The regression coefficients $\hat{\beta}_{x_{i} x_{j}}$ for each variable $x_{i}$ are obtained via a multiple regression analysis after an Arithmetic Mean Imputation (MEAN) is applied to the dataset. 


\section{NED KOCK}

An alternative to using Arithmetic Mean Imputation (MEAN), which tends to lead to an exacerbation of the biases and that is therefore not employed here, is to conduct the multiple regression analysis to obtain the regression coefficients $\hat{\beta}_{x_{i} x_{j}}$ after a listwise deletion. The use of deletion is particularly problematic here because the regression equation will typically have quite a few predictors, and thus a great deal of data may end up being lost after a listwise deletion.

\section{Hierarchical Regression Imputation}

The Hierarchical Regression Imputation (HREGR) method, a new method, assigns values to each missing element $\dot{x}_{i r}$ according to (3), where $k$ is the number of manifest variables used in a model, $N_{m}$ is the number of missing values in $x_{i}$, and each of the elements of the matrix of estimated correlations $\hat{\Sigma}_{x_{i} x_{j}}$ is calculated after a pairwise deletion of missing elements is conducted for each pair of variables $x_{i}$ and $x_{j}$. In this equation $\max \left(\hat{\Sigma}_{x_{i} x_{j}}\right)$ is the maximum estimated correlation between the manifest variable $x_{i}$ and any other manifest variable $x_{j}$ for which a corresponding non-missing value $x_{j r}$ exists.

$$
\begin{gathered}
\dot{x}_{i r}=\max \left(\hat{\Sigma}_{x_{i} x_{j}}\right) x_{j r}, \\
j=1 \ldots k, j \neq i, r=1 \ldots N_{m} .
\end{gathered}
$$

In the Hierarchical Regression Imputation (HREGR) method each missing element $\dot{x}_{i r}$ is replaced with the corresponding expected value of $x_{i}$ given a variable $x_{j}$, stored in column $j$ of the dataset, where $x_{j}$ is the variable with the highest correlation with $x_{i}$ after a pairwise deletion of missing elements.

A pairwise deletion is preferred over an Arithmetic Mean Imputation (MEAN) for the calculation of the correlations $\hat{\Sigma}_{x_{i} x_{j}}$ because it leads to less bias, as indicated by exploratory versions of this method that we developed and tested. In datasets with multiple variables and widespread missing data elements, pairwise deletions usually lead to much lesser amounts of data loss than listwise deletions. Nevertheless, the results of analyses conducted after pairwise deletions tend to be dependent on the pair-specific idiosyncrasies of missing data patterns. 


\section{Stochastic Multiple Regression Imputation}

The Stochastic Multiple Regression Imputation (MSREG) method assigns values to each missing element $\dot{x}_{i r}$ according to (4), where $k$ is the number of manifest variables used in a model, $N_{m}$ is the number of missing values in $x_{i}$, and $\operatorname{Srandn}(\ldots)$ is a function that returns a different element of a standardized normally distributed random column vector each time it is invoked.

$$
\begin{aligned}
& \dot{x}_{i r}=\sum_{j=1}^{k} \hat{\beta}_{x_{i} x_{j}} x_{j r}+\left(\sqrt{\left(1-\sum_{j=1}^{k} \hat{\beta}_{x_{i} x_{j}} \hat{\Sigma}_{x_{i} x_{j}}\right)}\right) \operatorname{Srandn}(\ldots ;), \\
& j=1 \ldots k, j \neq i, r=1 \ldots N_{m} .
\end{aligned}
$$

The Stochastic Multiple Regression Imputation (MSREG) method is similar to the Multiple Regression Imputation (MREGR) method. The key difference is that in this stochastic variety, implemented via the equation above, normal random error is added to the new values due to the assumption that not doing so can create a downward bias in standard errors. Such a bias could lead to an exacerbation of Type I errors. The random error elements yielded by $\operatorname{Srandn}(\cdots: \ldots)$ are weighted so that they collectively account for all of the variance in $x_{i}$ that is not explained by the predictors $x_{j}(j=1 \ldots k, j \neq i)$.

Although the above assumption regarding standard error bias may be a reasonable one with respect to standard multiple regression and covariance-based SEM, in PLS-SEM path coefficients tend to present downward biases even without missing data. Therefore, a downward bias in standard errors may compensate for the related decrease in statistical power, due to the downward path coefficient bias, in turn countering an exacerbation in Type II errors (and a reduction in power).

\section{Stochastic Hierarchical Regression Imputation}

The Stochastic Hierarchical Regression Imputation (HSREG) method, another new method, assigns values to each missing element $\dot{x}_{i r}$ according to (5), where $k$ is the number of manifest variables used in a model, $N_{m}$ is the number of missing values in $x_{i}$, and $\operatorname{Srandn}\left(\mathrm{H}_{\mathrm{i}}\right)$ is a function that returns a different element of a standardized normally distributed random column vector each time it is invoked. 


\section{NED KOCK}

$$
\begin{gathered}
\dot{x}_{i r}=\max \left(\hat{\Sigma}_{x_{i} x_{j}}\right) x_{j r}+\left(\sqrt{1-\max \left(\hat{\Sigma}_{x_{i} x_{j}}\right)^{2}}\right) \operatorname{Srandn}(\cdots \ldots), \\
j=1 \ldots k, j \neq i, r=1 \ldots N_{m} .
\end{gathered}
$$

The Stochastic Hierarchical Regression Imputation (HSREG) method is similar to the Hierarchical Regression Imputation (HREGR) method. The key difference (analogously to the discussion above) in this stochastic variety is that normal random error is added to the new values due to the assumption that not doing so can create a downward bias in standard errors and an overall deleterious effect on type I error rates. Although this assumption may find general application in standard multiple regression and covariance-based SEM, it may not readily apply to PLS-SEM.

\section{Monte Carlo Experiment}

A Monte Carlo experiment based on the true population model shown in Figure 2 was conducted to assess the performance of the five missing data imputation methods discussed in the previous section. Performance was assessed in terms of path coefficient bias and standard error inflation.

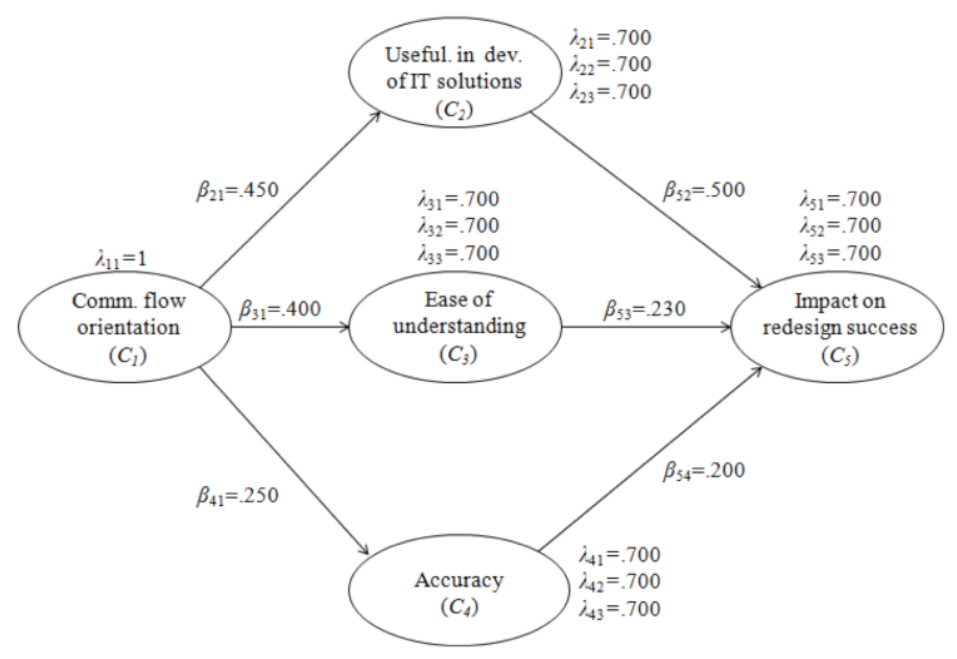

Figure 2. True population model 


\section{SINGLE MISSING DATA IMPUTATION IN PLS-BASED SEM}

When creating data for our Monte Carlo experiment we varied the following conditions: percentage of missing data $(0 \%, 30 \%, 40 \%$, and 50\%), and sample size $(100,300$, and 500). This led to a $4 \times 3$ factorial design, with 12 conditions, where 1,000 samples were analyzed for each of these 12 conditions for a total of 12,000 samples.

The PLS Mode A algorithm with the path weighting scheme (Lohmöller, 1989) was used in the analyses. These are the most widely used algorithm (PLS Mode A) and inner model estimation scheme (path weighting) in the context of PLS-SEM. Results were obtained for analyses with no missing data (NMD), Arithmetic Mean Imputation (MEAN), Multiple Regression Imputation (MREGR), Hierarchical Regression Imputation (HREGR), Stochastic Multiple Regression Imputation (MSREG), and Stochastic Hierarchical Regression Imputation (HSREG).

A summarized set of results are shown in Table 1 and Figure 3, where $N=300$ and $30 \%$ missing data (MAR). In the figure, consider the absolute path coefficient differences with respect to no missing data (NMD) estimates, to highlight the performance of the various missing data imputation methods. In the table, true path coefficients, mean path coefficient estimates, and standard errors of path coefficient estimates are shown next to one another. Full results, for all percentages of missing data and sample sizes included in the simulation, are available in Appendix A. Because all loadings are the same in the true population model, loading-related estimates for only one indicator of the composites are shown. This avoids crowding and repetition, as the same pattern of results repeats itself in connection with all loadings.

The mean path coefficient estimates that are shown underlined in the table were obtained through the application of the PLS Mode A algorithm to datasets where no data was missing (NMD). Note that they generally underestimate the true path coefficients. This underestimation stems from the use of composites in PLS-SEM, discussed earlier, which leads to an attenuation of composite correlations (Nunnally \& Bernstein, 1994). This correlation attenuation extends to the path coefficients (Kock, 2015b), leading to the observed underestimation. The opposite effect is observed in connection with loadings, which tend to be overestimated in PLS-SEM.

Multiple Regression Imputation (MREGR) yielded the least biased mean path coefficient estimates, followed by Arithmetic Mean Imputation (MEAN). When we look at mean loading estimates, Arithmetic Mean Imputation (MEAN) yielded the least biased results, followed by Stochastic Hierarchical Regression Imputation (HSREG) and Hierarchical Regression Imputation (HREGR). 


\section{NED KOCK}

Compared with the no missing data condition (NMD), none of the methods induced a significant bias in standard errors. This is noteworthy since prior results outside the context of PLS-SEM have tended to show a significant downward bias in standard errors, particularly for non-stochastic varieties. Such downward bias in standard errors has led to concerns regarding an inflation in type I errors, and warnings against the use of single missing data imputation methods in general (Enders, 2010; Newman, 2014).

Table 1. Summarized Monte Carlo experiment results $(N=300,30 \%$ MAR data)

\begin{tabular}{lcccccc} 
Missing data imputation scheme & NMD & MEAN & MREGR & HREGR & MSREG & HSREG \\
\hline CO>GT(TruePath) & 0.450 & 0.450 & 0.450 & 0.450 & 0.450 & 0.450 \\
CO>GT(AvgPath) & $\underline{0.390}$ & 0.348 & 0.367 & 0.354 & 0.333 & 0.300 \\
CO>GT(SEPath) & 0.075 & 0.113 & 0.110 & 0.113 & 0.138 & 0.162 \\
CO>EU(TruePath) & 0.400 & 0.400 & 0.400 & 0.400 & 0.400 & 0.400 \\
CO>EU(AvgPath) & $\underline{0.349}$ & 0.312 & 0.321 & 0.313 & 0.289 & 0.262 \\
CO>EU(SEPath) & 0.069 & 0.101 & 0.108 & 0.106 & 0.133 & 0.151 \\
CO>AC(TruePath) & 0.250 & 0.250 & 0.250 & 0.250 & 0.250 & 0.250 \\
CO>AC(AvgPath) & $\underline{0.219}$ & 0.198 & 0.206 & 0.195 & 0.188 & 0.161 \\
CO>AC(SEPath) & 0.062 & 0.078 & 0.090 & 0.083 & 0.100 & 0.108 \\
GT>SU(TruePath) & 0.500 & 0.500 & 0.500 & 0.500 & 0.500 & 0.500 \\
GT>SU(AvgPath) & $\underline{0.381}$ & 0.357 & 0.359 & 0.352 & 0.334 & 0.312 \\
GT>SU(SEPath) & 0.127 & 0.152 & 0.156 & 0.158 & 0.179 & 0.195 \\
EU>SU(TruePath) & 0.230 & 0.230 & 0.230 & 0.230 & 0.230 & 0.230 \\
EU>SU(AvgPath) & $\underline{0.192}$ & 0.183 & 0.199 & 0.178 & 0.188 & 0.163 \\
EU>SU(SEPath) & 0.062 & 0.072 & 0.077 & 0.078 & 0.082 & 0.089 \\
AC>SU(TruePath) & 0.200 & 0.200 & 0.200 & 0.200 & 0.200 & 0.200 \\
AC>SU(AvgPath) & $\underline{0.165}$ & 0.157 & 0.176 & 0.154 & 0.166 & 0.141 \\
AC>SU(SEPath) & 0.058 & 0.067 & 0.073 & 0.072 & 0.077 & 0.081 \\
GT3<GT(TrueLoad) & 0.700 & 0.700 & 0.700 & 0.700 & 0.700 & 0.700 \\
GT3<GT(AvgLoad) & $\underline{0.811}$ & 0.691 & 0.606 & 0.649 & 0.623 & 0.652 \\
GT3<GT(SELoad) & 0.113 & 0.042 & 0.120 & 0.076 & 0.115 & 0.090 \\
\hline
\end{tabular}

Notes: NMD = no missing data; MEAN = Arithmetic Mean Imputation; MREGR = Multiple Regression Imputation; HREGR = Hierarchical Regression Imputation; MSREG = Stochastic Multiple Regression Imputation; HSREG = Stochastic Hierarchical Regression Imputation; $X X>Y Y=$ link from composite $X X$ to $Y Y$; $\mathrm{CO}=$ communication flow orientation $\left(C_{1}\right)$; GT = usefulness in the development of IT solutions $\left(C_{2}\right)$; EU = ease of understanding $\left(C_{3}\right) ; \mathrm{AC}=$ accuracy $\left(C_{4}\right)$; $\mathrm{SU}=$ impact on redesign success $\left(C_{5}\right)$; TruePath $=$ true path coefficient; AvgPath = mean path coefficient estimate; SEPath = standard error of path coefficient estimate; TrueLoad = true loading; AvgLoad = mean loading estimate; SELoad = standard error of loading estimate. 


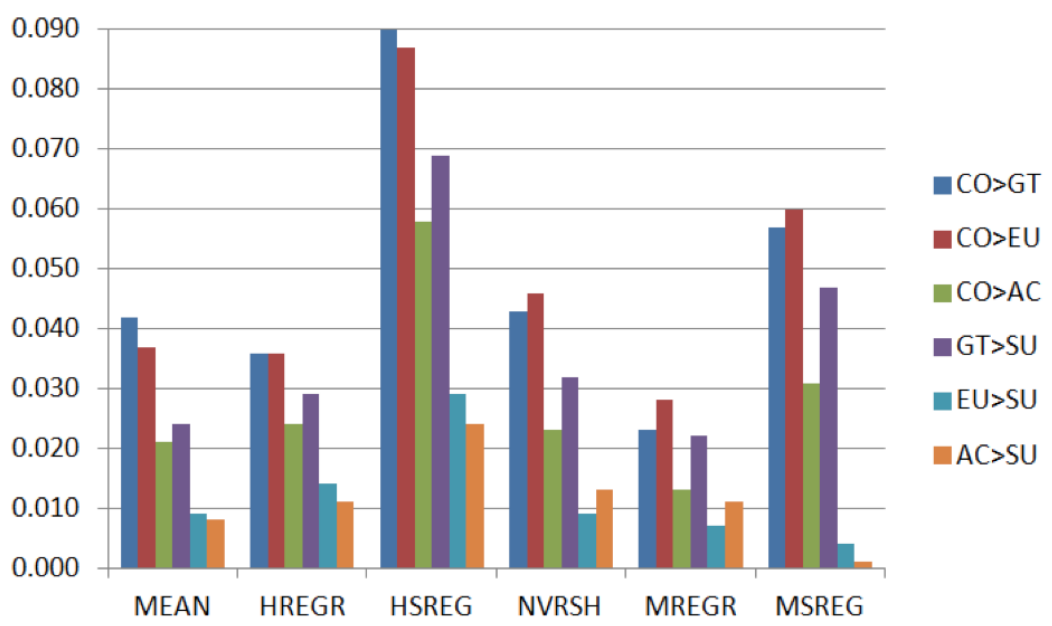

Figure 3. Absolute path coefficient differences with respect to no missing data (NMD) estimates

\section{Empirical Illustration}

Summarized in Table 2 are results of an empirical field study related to the illustrative and true population models discussed earlier. It served as the basis for the development of the illustrative and true population models. Shown next to one another are estimated path coefficients (top part of the table), and loadings (bottom part of the table). All path coefficients and loadings are shown. Except for the column "NMD", all other columns show results with $30 \%$ missing data (MAR).

The data for this empirical study was collected from 156 individuals who participated in various business process redesign projects in organizations located in Northeastern U.S.A. The participants employed one of two business process modeling approaches. One of the modeling approaches focused primarily on the communication flow within business processes. The other focused primarily on the chronological flow of activities. Both approaches are illustrated in Appendix B. Appendix $\mathrm{C}$ has the questionnaire used for data collection.

Overall, all missing data imputation methods analyzed yielded estimates consistent with communication flow optimization theory (Kock, 2003). No method led to biases that were severe enough, at $30 \%$ missing data, to generate non-significant $P$ values. Given this, we could say that the empirical study results provide real data validation of all imputation methods, and to a certain extend 


\section{NED KOCK}

qualified support for all of them. This is because the theory, which forms the underlying theoretical foundation for the model, has been validated before in multiple empirical studies employing different datasets and methods (DaneshPajou, 2005; Danesh-Pajou \& Kock, 2005; Kock et al., 2008; 2009).

Table 2. Empirical study results

\begin{tabular}{|c|c|c|c|c|c|c|}
\hline Missing data imputation scheme & NMD & MEAN & HREGR & HSREG & MREGR & MSREG \\
\hline $\mathrm{CO}>\mathrm{GT}$ & $0.485^{\mathrm{a}}$ & $0.427^{a}$ & $0.472^{\mathrm{a}}$ & $0.445^{\mathrm{a}}$ & $0.462^{\mathrm{a}}$ & $0.379^{a}$ \\
\hline $\mathrm{CO}>\mathrm{EU}$ & $0.362^{\mathrm{a}}$ & $0.244^{\mathrm{a}}$ & $0.282^{\mathrm{a}}$ & $0.313^{a}$ & $0.248^{a}$ & $0.263^{a}$ \\
\hline$C O>A C$ & $0.269^{a}$ & $0.184^{\mathrm{b}}$ & $0.209^{b}$ & $0.183^{b}$ & $0.195^{b}$ & $0.213^{b}$ \\
\hline GT>SU & $0.506^{a}$ & $0.531^{a}$ & $0.536^{a}$ & $0.527^{a}$ & $0.532^{\mathrm{a}}$ & $0.493^{a}$ \\
\hline EU>SU & $0.217^{\mathrm{b}}$ & $0.184^{\mathrm{b}}$ & $0.204^{b}$ & $0.233^{b}$ & $0.187^{b}$ & $0.174^{\mathrm{c}}$ \\
\hline$A C>S U$ & $0.194^{\mathrm{b}}$ & $0.181^{b}$ & $0.150^{c}$ & $0.146^{c}$ & $0.173^{c}$ & $0.170^{c}$ \\
\hline $\mathrm{GT} 1<\mathrm{GT}$ & 0.926 & 0.854 & 0.938 & 0.883 & 0.899 & 0.900 \\
\hline $\mathrm{GT} 2<\mathrm{GT}$ & 0.880 & 0.883 & 0.919 & 0.887 & 0.897 & 0.863 \\
\hline $\mathrm{GT} 3<\mathrm{GT}$ & 0.893 & 0.878 & 0.929 & 0.885 & 0.907 & 0.855 \\
\hline EU1<EU & 0.796 & 0.740 & 0.815 & 0.801 & 0.786 & 0.742 \\
\hline EU2<EU & 0.875 & 0.831 & 0.853 & 0.816 & 0.862 & 0.827 \\
\hline EU3<EU & 0.910 & 0.884 & 0.909 & 0.901 & 0.903 & 0.871 \\
\hline$A C 1<A C$ & 0.916 & 0.926 & 0.925 & 0.918 & 0.926 & 0.926 \\
\hline$A C 2<A C$ & 0.868 & 0.812 & 0.863 & 0.847 & 0.840 & 0.794 \\
\hline$A C 3<A C$ & 0.753 & 0.674 & 0.723 & 0.634 & 0.703 & 0.677 \\
\hline SU1<SU & 0.937 & 0.914 & 0.950 & 0.913 & 0.934 & 0.895 \\
\hline SU2<SU & 0.947 & 0.934 & 0.957 & 0.916 & 0.949 & 0.919 \\
\hline SU3<SU & 0.932 & 0.913 & 0.944 & 0.925 & 0.933 & 0.908 \\
\hline
\end{tabular}

Notes: $N=156 ;{ }^{\mathrm{a}} P<.001,{ }^{\mathrm{b}} P<.01,{ }^{\mathrm{c}} P<.05$; PLS algorithm used $=\mathrm{PLS}$ Mode $\mathrm{A} ; P$ values calculated via bootstrapping with 500 resamples; NMD = no missing data; MEAN = Arithmetic Mean Imputation; MREGR = Multiple Regression Imputation; HREGR = Hierarchical Regression Imputation; MSREG = Stochastic Multiple Regression Imputation; HSREG = Stochastic Hierarchical Regression Imputation; $X X>Y Y=$ link from variable $\mathrm{XX}$ to $\mathrm{YY} ; \mathrm{CO}=$ communication flow orientation $\left(C_{1}\right)$; GT = usefulness in the development of IT solutions $\left(C_{2}\right)$; $\mathrm{EU}=$ ease of understanding $\left(C_{3}\right) ; \mathrm{AC}=$ accuracy $\left(C_{4}\right) ; \mathrm{SU}=$ impact on redesign success $\left(C_{5}\right)$;

$\mathrm{XX1} \ldots \mathrm{XX} n=$ indicators associated with composite $\mathrm{XX}$.

\section{Conclusion}

Multiple Regression Imputation (MREGR) yielded the least biased mean path coefficient estimates, followed by Arithmetic Mean Imputation (MEAN). With respect to mean loading estimates, Arithmetic Mean Imputation (MEAN) yielded the least biased results, followed by Stochastic Hierarchical Regression Imputation (HSREG) and Hierarchical Regression Imputation (HREGR). 


\section{SINGLE MISSING DATA IMPUTATION IN PLS-BASED SEM}

None of the methods induced a significant bias in standard errors when compared with the no missing data condition (NMD). This is at odds with past results outside the context of PLS-SEM, which tended to show a significant downward bias in standard errors, particularly for non-stochastic imputation methods. This observed downward bias in standard errors has led to concerns regarding type I error inflation, and admonitions against the use of single missing data imputation methods in general. PLS-SEM may be a fertile ground for the application of single missing data imputation methods, although more research is needed to shed light as to whether this is truly the case and why.

\section{References}

Akter, S., Fosso Wamba, S., \& Dewan, S. (2017). Why PLS-SEM is suitable for complex modelling? An empirical illustration in big data analytics quality. Production Planning \& Control, 28(11-12), 1011-1021. doi: 10.1080/09537287.2016.1267411

Danesh-Pajou, A. (2005). IT-enabled process redesign: Using communication flow optimization theory in an information intensive environment. Doctoral Dissertation. Philadelphia, PA: Temple University.

Danesh-Pajou, A., \& Kock, N. (2005). An experimental study of process representation approaches and their impact on perceived modeling quality and redesign success. Business Process Management Journal, 11(6), 724-735. doi: 10.1108/14637150510630882

Enders, C. K. (2010). Applied missing data analysis. New York, NY: The Guilford Press.

Kline, R. B. (2010). Principles and practice of structural equation modeling. New York, NY: The Guilford Press.

Kock, N. (2003). Communication-focused business process redesign: Assessing a communication flow optimization model through an action research study at a defense contractor. IEEE Transactions on Professional Communication, 46(1), 35-54. doi: 10.1109/tpc.2002.808350

Kock, N. (2007). Systems analysis and design fundamentals: A business process redesign approach. Thousand Oaks, CA: Sage Publications. doi: $10.4135 / 9781452224794$ 


\section{NED KOCK}

Kock, N. (2015a). One-tailed or two-tailed P values in PLS-SEM? International Journal of e-Collaboration, 11(2), 1-7. doi:

10.4018/ijec.2015040101

Kock, N. (2015b). A note on how to conduct a factor-based PLS-SEM analysis. International Journal of e-Collaboration, 11(3), 1-9. doi:

10.4018/ijec.2015070101

Kock, N. (2016). Non-normality propagation among latent variables and indicators in PLS-SEM simulations. Journal of Modern Applied Statistical Methods, 15(1), 299-315. doi: 10.22237/jmasm/1462076100

Kock, N., Danesh-Pajou, A., \& Komiak, P. (2008). A discussion and test of a communication flow optimization approach for business process redesign. Knowledge and Process Management, 15(1), 72-85. doi: 10.1002/kpm.301

Kock, N., Verville, J., Danesh-Pajou, A., \& DeLuca, D. (2009). Communication flow orientation in business process modeling and its effect on redesign success: Results from a field study. Decision Support Systems, 46(2), 562-575. doi: 10.1016/j.dss.2008.10.002

Lohmöller, J.-B. (1989). Latent variable path modeling with partial least squares. Heidelberg, Germany: Physica-Verlag. doi: 10.1007/978-3-642-52512-4

Mendling, J., Strembeck, M., \& Recker, J. (2012). Factors of process model comprehension-Findings from a series of experiments. Decision Support Systems, 53(1), 195-206. doi: 10.1016/j.dss.2011.12.013

Newman, D. A. (2014). Missing data: Five practical guidelines. Organizational Research Methods, 17(4), 372-411. doi: $10.1177 / 1094428114548590$

Nunnally, J. C., \& Bernstein, I. H. (1994). Psychometric theory. New York, NY: McGraw-Hill.

Rigdon, E. E. (2016). Choosing PLS path modeling as analytical method in European management research: A realist perspective. European Management Journal, 34(6), 598-605. doi: 10.1016/j.emj.2016.05.006

Wilkinson, L. (1999). Statistical methods in psychology journals: Guidelines and explanations. American psychologist, 54(8), 594-604. doi: 10.1037/0003066x.54.8.594

Wright, S. (1934). The method of path coefficients. The Annals of Mathematical Statistics, 5(3), 161-215. doi: 10.1214/aoms/1177732676

Wright, S. (1960). Path coefficients and path regressions: Alternative or complementary concepts? Biometrics, 16(2), 189-202. doi: 10.2307/2527551 


\section{Appendix A: Full Monte Carlo Experiment Results}

The full Monte Carlo experiment results are provided in the tables below. Notes: $\mathrm{NMD}=$ no missing data; MEAN = Arithmetic Mean Imputation; MREGR = Multiple Regression Imputation; HREGR = Hierarchical Regression Imputation; MSREG = Stochastic Multiple Regression Imputation; HSREG = Stochastic Hierarchical Regression Imputation; $\mathrm{XX}>\mathrm{YY}=$ link from composite $\mathrm{XX}$ to $\mathrm{YY}$; $\mathrm{CO}=$ communication flow orientation $\left(C_{1}\right)$; GT $=$ usefulness in the development of IT solutions $\left(C_{2}\right)$; EU = ease of understanding $\left(C_{3}\right)$; $\mathrm{AC}=\operatorname{accuracy}\left(C_{4}\right)$; $\mathrm{SU}=$ impact on redesign success $\left(C_{5}\right)$; TruePath $=$ true path coefficient; AvgPath $=$ mean path coefficient estimate; SEPath = standard error of estimate; TrueLoad = true loading; AvgLoad = mean loading estimate; SELoad $=$ standard error of estimate.

Table A1a. Monte Carlo experiment results with a sample size of 100 and $30 \%$ missing data.

\begin{tabular}{lc|ccccc} 
Missing data imputation scheme & NMD & MEAN & MREGR & HREGR & MSREG & HSREG \\
\hline CO>GT(TruePath) & 0.450 & 0.450 & 0.450 & 0.450 & 0.450 & 0.450 \\
CO>GT(AvgPath) & 0.394 & 0.354 & 0.364 & 0.308 & 0.362 & 0.327 \\
CO>GT(SEPath) & 0.094 & 0.129 & 0.133 & 0.175 & 0.148 & 0.171 \\
CO>EU(TruePath) & 0.400 & 0.400 & 0.400 & 0.400 & 0.400 & 0.400 \\
CO>EU(AvgPath) & 0.355 & 0.323 & 0.326 & 0.280 & 0.335 & 0.308 \\
CO>EU(SEPath) & 0.096 & 0.120 & 0.130 & 0.161 & 0.145 & 0.156 \\
CO>AC(TruePath) & 0.250 & 0.250 & 0.250 & 0.250 & 0.250 & 0.250 \\
CO>AC(AvgPath) & 0.227 & 0.205 & 0.205 & 0.172 & 0.214 & 0.196 \\
CO>AC(SEPath) & 0.093 & 0.111 & 0.124 & 0.140 & 0.148 & 0.153 \\
GT>SU(TruePath) & 0.500 & 0.500 & 0.500 & 0.500 & 0.500 & 0.500 \\
GT>SU(AvgPath) & 0.384 & 0.355 & 0.353 & 0.319 & 0.351 & 0.328 \\
GT>SU(SEPath) & 0.141 & 0.170 & 0.178 & 0.206 & 0.188 & 0.206 \\
EU>SU(TruePath) & 0.230 & 0.230 & 0.230 & 0.230 & 0.230 & 0.230 \\
EU>SU(AvgPath) & 0.193 & 0.188 & 0.187 & 0.172 & 0.207 & 0.196 \\
EU>SU(SEPath) & 0.094 & 0.103 & 0.112 & 0.121 & 0.121 & 0.129 \\
AC>SU(TruePath) & 0.200 & 0.200 & 0.200 & 0.200 & 0.200 & 0.200 \\
AC>SU(AvgPath) & 0.172 & 0.165 & 0.167 & 0.150 & 0.193 & 0.183 \\
AC>SU(SEPath) & 0.091 & 0.107 & 0.114 & 0.123 & 0.130 & 0.134 \\
GT3<GT(TrueLoad) & 0.700 & 0.700 & 0.700 & 0.700 & 0.700 & 0.700 \\
GT3<GT(AvgLoad) & 0.810 & 0.687 & 0.645 & 0.644 & 0.593 & 0.603 \\
GT3<GT(SELoad) & 0.118 & 0.072 & 0.105 & 0.128 & 0.156 & 0.165 \\
\hline & & & & &
\end{tabular}




\section{NED KOCK}

Table A1b. Monte Carlo experiment results with a sample size of 100 and $40 \%$ missing data.

\begin{tabular}{|c|c|c|c|c|c|c|}
\hline Missing data imputation scheme & NMD & MEAN & MREGR & HREGR & MSREG & HSREG \\
\hline CO>GT(TruePath) & 0.450 & 0.450 & 0.450 & 0.450 & 0.450 & 0.450 \\
\hline $\mathrm{CO}>\mathrm{GT}($ AvgPath) & 0.394 & 0.309 & 0.315 & 0.247 & 0.307 & 0.264 \\
\hline CO>GT(SEPath) & 0.094 & 0.188 & 0.193 & 0.240 & 0.223 & 0.251 \\
\hline $\mathrm{CO}>\mathrm{EU}$ (TruePath) & 0.400 & 0.400 & 0.400 & 0.400 & 0.400 & 0.400 \\
\hline CO>EU(AvgPath) & 0.355 & 0.280 & 0.283 & 0.225 & 0.275 & 0.240 \\
\hline CO>EU(SEPath) & 0.096 & 0.185 & 0.194 & 0.226 & 0.219 & 0.239 \\
\hline $\mathrm{CO}>\mathrm{AC}$ (TruePath) & 0.250 & 0.250 & 0.250 & 0.250 & 0.250 & 0.250 \\
\hline $\mathrm{CO}>\mathrm{AC}($ AvgPath) & 0.227 & 0.186 & 0.182 & 0.145 & 0.189 & 0.165 \\
\hline $\mathrm{CO}>\mathrm{AC}(\mathrm{SEP}$ ath) & 0.093 & 0.170 & 0.188 & 0.185 & 0.208 & 0.211 \\
\hline GT>SU(TruePath) & 0.500 & 0.500 & 0.500 & 0.500 & 0.500 & 0.500 \\
\hline GT>SU(AvgPath) & 0.384 & 0.320 & 0.324 & 0.272 & 0.311 & 0.280 \\
\hline GT>SU(SEPath) & 0.141 & 0.222 & 0.227 & 0.263 & 0.246 & 0.270 \\
\hline EU>SU(TruePath) & 0.230 & 0.230 & 0.230 & 0.230 & 0.230 & 0.230 \\
\hline EU>SU(AvgPath) & 0.193 & 0.191 & 0.189 & 0.163 & 0.189 & 0.178 \\
\hline EU>SU(SEPath) & 0.094 & 0.144 & 0.157 & 0.163 & 0.186 & 0.195 \\
\hline AC>SU(TruePath) & 0.200 & 0.200 & 0.200 & 0.200 & 0.200 & 0.200 \\
\hline AC>SU(AvgPath) & 0.172 & 0.177 & 0.177 & 0.146 & 0.186 & 0.164 \\
\hline AC>SU(SEPath) & 0.091 & 0.157 & 0.172 & 0.170 & 0.204 & 0.208 \\
\hline GT3<GT(TrueLoad) & 0.700 & 0.700 & 0.700 & 0.700 & 0.700 & 0.700 \\
\hline GT3<GT(AvgLoad) & 0.810 & 0.479 & 0.440 & 0.444 & 0.395 & 0.398 \\
\hline GT3<GT(SELoad) & 0.118 & 0.261 & 0.295 & 0.306 & 0.347 & 0.359 \\
\hline
\end{tabular}

Table A1c. Monte Carlo experiment results with a sample size of 100 and $50 \%$ missing data.

\begin{tabular}{lc|ccccc} 
Missing data imputation scheme & NMD & MEAN & MREGR & HREGR & MSREG & HSREG \\
\hline CO>GT(TruePath) & 0.450 & 0.450 & 0.450 & 0.450 & 0.450 & 0.450 \\
CO>GT(AvgPath) & 0.394 & 0.241 & 0.248 & 0.170 & 0.227 & 0.183 \\
CO>GT(SEPath) & 0.094 & 0.272 & 0.287 & 0.327 & 0.323 & 0.345 \\
CO>EU(TruePath) & 0.400 & 0.400 & 0.400 & 0.400 & 0.400 & 0.400 \\
CO>EU(AvgPath) & 0.355 & 0.215 & 0.211 & 0.145 & 0.190 & 0.159 \\
CO>EU(SEPath) & 0.096 & 0.263 & 0.284 & 0.308 & 0.323 & 0.327 \\
CO>AC(TruePath) & 0.250 & 0.250 & 0.250 & 0.250 & 0.250 & 0.250 \\
CO>AC(AvgPath) & 0.227 & 0.146 & 0.151 & 0.110 & 0.136 & 0.113 \\
CO>AC(SEPath) & 0.093 & 0.227 & 0.242 & 0.228 & 0.276 & 0.270 \\
GT>SU(TruePath) & 0.500 & 0.500 & 0.500 & 0.500 & 0.500 & 0.500 \\
GT>SU(AvgPath) & 0.384 & 0.267 & 0.263 & 0.208 & 0.238 & 0.207 \\
GT>SU(SEPath) & 0.141 & 0.292 & 0.303 & 0.337 & 0.351 & 0.359 \\
EU>SU(TruePath) & 0.230 & 0.230 & 0.230 & 0.230 & 0.230 & 0.230 \\
EU>SU(AvgPath) & 0.193 & 0.172 & 0.168 & 0.137 & 0.163 & 0.139 \\
EU>SU(SEPath) & 0.094 & 0.212 & 0.239 & 0.213 & 0.264 & 0.259 \\
AC>SU(TruePath) & 0.200 & 0.200 & 0.200 & 0.200 & 0.200 & 0.200 \\
AC>SU(AvgPath) & 0.172 & 0.152 & 0.149 & 0.118 & 0.153 & 0.135 \\
AC>SU(SEPath) & 0.091 & 0.219 & 0.242 & 0.213 & 0.270 & 0.263 \\
GT3<GT(TrueLoad) & 0.700 & 0.700 & 0.700 & 0.700 & 0.700 & 0.700 \\
GT3<GT(AvgLoad) & 0.810 & 0.284 & 0.250 & 0.263 & 0.217 & 0.214 \\
GT3<GT(SELoad) & 0.118 & 0.451 & 0.480 & 0.483 & 0.511 & 0.526 \\
\hline & & & & &
\end{tabular}




\section{SINGLE MISSING DATA IMPUTATION IN PLS-BASED SEM}

Table A2a. Monte Carlo experiment results with a sample size of 300 and $30 \%$ missing data.

\begin{tabular}{|c|c|c|c|c|c|c|}
\hline Missing data imputation scheme & NMD & MEAN & MREGR & HREGR & MSREG & HSREG \\
\hline CO>GT(TruePath) & 0.450 & 0.450 & 0.450 & 0.450 & 0.450 & 0.450 \\
\hline $\mathrm{CO}>\mathrm{GT}($ AvgPath) & 0.390 & 0.348 & 0.354 & 0.300 & 0.367 & 0.333 \\
\hline CO>GT(SEPath) & 0.075 & 0.113 & 0.113 & 0.162 & 0.110 & 0.138 \\
\hline $\mathrm{CO}>\mathrm{EU}$ (TruePath) & 0.400 & 0.400 & 0.400 & 0.400 & 0.400 & 0.400 \\
\hline $\mathrm{CO}>\mathrm{EU}($ AvgPath) & 0.349 & 0.312 & 0.313 & 0.262 & 0.321 & 0.289 \\
\hline CO>EU(SEPath) & 0.069 & 0.101 & 0.106 & 0.151 & 0.108 & 0.133 \\
\hline $\mathrm{CO}>\mathrm{AC}$ (TruePath) & 0.250 & 0.250 & 0.250 & 0.250 & 0.250 & 0.250 \\
\hline $\mathrm{CO}>\mathrm{AC}($ AvgPath $)$ & 0.219 & 0.198 & 0.195 & 0.161 & 0.206 & 0.188 \\
\hline CO>AC(SEPath) & 0.062 & 0.078 & 0.083 & 0.108 & 0.090 & 0.100 \\
\hline GT>SU(TruePath) & 0.500 & 0.500 & 0.500 & 0.500 & 0.500 & 0.500 \\
\hline GT>SU(AvgPath) & 0.381 & 0.357 & 0.352 & 0.312 & 0.359 & 0.334 \\
\hline GT>SU(SEPath) & 0.127 & 0.152 & 0.158 & 0.195 & 0.156 & 0.179 \\
\hline EU>SU(TruePath) & 0.230 & 0.230 & 0.230 & 0.230 & 0.230 & 0.230 \\
\hline EU>SU(AvgPath) & 0.192 & 0.183 & 0.178 & 0.163 & 0.199 & 0.188 \\
\hline EU>SU(SEPath) & 0.062 & 0.072 & 0.078 & 0.089 & 0.077 & 0.082 \\
\hline AC>SU(TruePath) & 0.200 & 0.200 & 0.200 & 0.200 & 0.200 & 0.200 \\
\hline AC>SU(AvgPath) & 0.165 & 0.157 & 0.154 & 0.141 & 0.176 & 0.166 \\
\hline AC>SU(SEPath) & 0.058 & 0.067 & 0.072 & 0.081 & 0.073 & 0.077 \\
\hline GT3<GT(TrueLoad) & 0.700 & 0.700 & 0.700 & 0.700 & 0.700 & 0.700 \\
\hline GT3<GT(AvgLoad) & 0.811 & 0.691 & 0.649 & 0.652 & 0.606 & 0.623 \\
\hline GT3<GT(SELoad) & 0.113 & 0.042 & 0.076 & 0.090 & 0.120 & 0.115 \\
\hline
\end{tabular}

Table A2b. Monte Carlo experiment results with a sample size of 300 and $40 \%$ missing data.

\begin{tabular}{lc|ccccc} 
Missing data imputation scheme & NMD & MEAN & MREGR & HREGR & MSREG & HSREG \\
\hline CO>GT(TruePath) & 0.450 & 0.450 & 0.450 & 0.450 & 0.450 & 0.450 \\
CO>GT(AvgPath) & 0.390 & 0.309 & 0.311 & 0.240 & 0.308 & 0.264 \\
CO>GT(SEPath) & 0.075 & 0.160 & 0.165 & 0.224 & 0.173 & 0.209 \\
CO>EU(TruePath) & 0.400 & 0.400 & 0.400 & 0.400 & 0.400 & 0.400 \\
CO>EU(AvgPath) & 0.349 & 0.273 & 0.274 & 0.211 & 0.271 & 0.234 \\
CO>EU(SEPath) & 0.069 & 0.147 & 0.152 & 0.204 & 0.162 & 0.191 \\
CO>AC(TruePath) & 0.250 & 0.250 & 0.250 & 0.250 & 0.250 & 0.250 \\
CO>AC(AvgPath) & 0.219 & 0.176 & 0.174 & 0.132 & 0.178 & 0.156 \\
CO>AC(SEPath) & 0.062 & 0.113 & 0.116 & 0.142 & 0.129 & 0.138 \\
GT>SU(TruePath) & 0.500 & 0.500 & 0.500 & 0.500 & 0.500 & 0.500 \\
GT>SU(AvgPath) & 0.381 & 0.323 & 0.320 & 0.264 & 0.314 & 0.282 \\
GT>SU(SEPath) & 0.127 & 0.191 & 0.196 & 0.246 & 0.207 & 0.235 \\
EU>SU(TruePath) & 0.230 & 0.230 & 0.230 & 0.230 & 0.230 & 0.230 \\
EU>SU(AvgPath) & 0.192 & 0.186 & 0.180 & 0.157 & 0.201 & 0.184 \\
EU>SU(SEPath) & 0.062 & 0.087 & 0.094 & 0.101 & 0.096 \\
AC>SU(TruePath) & 0.200 & 0.200 & 0.200 & 0.200 & 0.200 \\
AC>SU(AvgPath) & 0.165 & 0.161 & 0.161 & 0.138 & 0.180 & 0.099 \\
AC>SU(SEPath) & 0.058 & 0.083 & 0.085 & 0.097 & 0.099 & 0.103 \\
GT3<GT(TrueLoad) & 0.700 & 0.700 & 0.700 & 0.700 & 0.700 & 0.700 \\
GT3<GT(AvgLoad) & 0.811 & 0.496 & 0.461 & 0.475 & 0.423 & 0.440 \\
GT3<GT(SELoad) & 0.113 & 0.221 & 0.256 & 0.253 & 0.296 & 0.286 \\
\hline & & & & &
\end{tabular}




\section{NED KOCK}

Table A2c. Monte Carlo experiment results with a sample size of 300 and $50 \%$ missing data.

\begin{tabular}{|c|c|c|c|c|c|c|}
\hline Missing data imputation scheme & NMD & MEAN & MREGR & HREGR & MSREG & HSREG \\
\hline CO>GT(TruePath) & 0.450 & 0.450 & 0.450 & 0.450 & 0.450 & 0.450 \\
\hline $\mathrm{CO}>\mathrm{GT}($ AvgPath) & 0.390 & 0.243 & 0.252 & 0.176 & 0.243 & 0.193 \\
\hline CO>GT(SEPath) & 0.075 & 0.229 & 0.226 & 0.288 & 0.246 & 0.284 \\
\hline CO>EU(TruePath) & 0.400 & 0.400 & 0.400 & 0.400 & 0.400 & 0.400 \\
\hline CO>EU(AvgPath) & 0.349 & 0.217 & 0.223 & 0.152 & 0.213 & 0.172 \\
\hline CO>EU(SEPath) & 0.069 & 0.209 & 0.208 & 0.264 & 0.230 & 0.260 \\
\hline $\mathrm{CO}>\mathrm{AC}($ TruePath) & 0.250 & 0.250 & 0.250 & 0.250 & 0.250 & 0.250 \\
\hline$C O>A C($ AvgPath) & 0.219 & 0.145 & 0.150 & 0.099 & 0.143 & 0.112 \\
\hline CO>AC(SEPath) & 0.062 & 0.150 & 0.154 & 0.179 & 0.180 & 0.194 \\
\hline GT>SU(TruePath) & 0.500 & 0.500 & 0.500 & 0.500 & 0.500 & 0.500 \\
\hline GT>SU(AvgPath) & 0.381 & 0.271 & 0.273 & 0.212 & 0.264 & 0.227 \\
\hline GT>SU(SEPath) & 0.127 & 0.246 & 0.249 & 0.300 & 0.263 & 0.295 \\
\hline EU>SU(TruePath) & 0.230 & 0.230 & 0.230 & 0.230 & 0.230 & 0.230 \\
\hline$E U>S U(A v g P a t h)$ & 0.192 & 0.183 & 0.185 & 0.143 & 0.194 & 0.168 \\
\hline EU>SU(SEPath) & 0.062 & 0.104 & 0.114 & 0.130 & 0.134 & 0.138 \\
\hline AC>SU(TruePath) & 0.200 & 0.200 & 0.200 & 0.200 & 0.200 & 0.200 \\
\hline$A C>S U(A v g P a t h)$ & 0.165 & 0.160 & 0.159 & 0.126 & 0.171 & 0.151 \\
\hline AC>SU(SEPath) & 0.058 & 0.112 & 0.123 & 0.124 & 0.141 & 0.137 \\
\hline GT3<GT(TrueLoad) & 0.700 & 0.700 & 0.700 & 0.700 & 0.700 & 0.700 \\
\hline GT3<GT(AvgLoad) & 0.811 & 0.329 & 0.296 & 0.311 & 0.256 & 0.268 \\
\hline GT3<GT(SELoad) & 0.113 & 0.386 & 0.417 & 0.412 & 0.456 & 0.453 \\
\hline
\end{tabular}

Table A3a. Monte Carlo experiment results with a sample size of 500 and $30 \%$ missing data.

\begin{tabular}{|c|c|c|c|c|c|c|}
\hline Missing data imputation scheme & NMD & MEAN & MREGR & HREGR & MSREG & HSREG \\
\hline $\mathrm{CO}>\mathrm{GT}$ (TruePath) & 0.450 & 0.450 & 0.450 & 0.450 & 0.450 & 0.450 \\
\hline CO>GT(AvgPath) & 0.389 & 0.346 & 0.352 & 0.296 & 0.363 & 0.328 \\
\hline CO>GT(SEPath) & 0.070 & 0.110 & 0.109 & 0.162 & 0.104 & 0.135 \\
\hline $\mathrm{CO}>\mathrm{EU}$ (TruePath) & 0.400 & 0.400 & 0.400 & 0.400 & 0.400 & 0.400 \\
\hline $\mathrm{CO}>\mathrm{EU}($ AvgPath) & 0.343 & 0.308 & 0.309 & 0.258 & 0.317 & 0.286 \\
\hline CO>EU(SEPath) & 0.067 & 0.100 & 0.102 & 0.149 & 0.102 & 0.129 \\
\hline $\mathrm{CO}>\mathrm{AC}($ TruePath $)$ & 0.250 & 0.250 & 0.250 & 0.250 & 0.250 & 0.250 \\
\hline $\mathrm{CO}>\mathrm{AC}$ (AvgPath) & 0.219 & 0.197 & 0.192 & 0.159 & 0.204 & 0.183 \\
\hline $\mathrm{CO}>\mathrm{AC}$ (SEPath) & 0.052 & 0.070 & 0.077 & 0.103 & 0.077 & 0.090 \\
\hline GT>SU(TruePath) & 0.500 & 0.500 & 0.500 & 0.500 & 0.500 & 0.500 \\
\hline GT>SU(AvgPath) & 0.380 & 0.354 & 0.348 & 0.309 & 0.358 & 0.333 \\
\hline GT>SU(SEPath) & 0.124 & 0.151 & 0.157 & 0.196 & 0.151 & 0.175 \\
\hline EU>SU(TruePath) & 0.230 & 0.230 & 0.230 & 0.230 & 0.230 & 0.230 \\
\hline EU>SU(AvgPath) & 0.189 & 0.180 & 0.176 & 0.160 & 0.198 & 0.184 \\
\hline EU>SU(SEPath) & 0.055 & 0.064 & 0.070 & 0.083 & 0.065 & 0.073 \\
\hline AC>SU(TruePath) & 0.200 & 0.200 & 0.200 & 0.200 & 0.200 & 0.200 \\
\hline$A C>S U(A v g P a t h)$ & 0.164 & 0.154 & 0.151 & 0.137 & 0.174 & 0.164 \\
\hline AC>SU(SEPath) & 0.054 & 0.063 & 0.067 & 0.077 & 0.061 & 0.067 \\
\hline GT3<GT(TrueLoad) & 0.700 & 0.700 & 0.700 & 0.700 & 0.700 & 0.700 \\
\hline GT3<GT(AvgLoad) & 0.811 & 0.692 & 0.652 & 0.654 & 0.609 & 0.627 \\
\hline GT3<GT(SELoad) & 0.113 & 0.035 & 0.069 & 0.082 & 0.113 & 0.106 \\
\hline
\end{tabular}




\section{SINGLE MISSING DATA IMPUTATION IN PLS-BASED SEM}

Table A3b. Monte Carlo experiment results with a sample size of 500 and $40 \%$ missing data.

\begin{tabular}{|c|c|c|c|c|c|c|}
\hline Missing data imputation scheme & NMD & MEAN & MREGR & HREGR & MSREG & HSREG \\
\hline CO>GT(TruePath) & 0.450 & 0.450 & 0.450 & 0.450 & 0.450 & 0.450 \\
\hline CO>GT(AvgPath) & 0.389 & 0.307 & 0.308 & 0.236 & 0.307 & 0.265 \\
\hline CO>GT(SEPath) & 0.070 & 0.155 & 0.158 & 0.223 & 0.164 & 0.201 \\
\hline CO>EU(TruePath) & 0.400 & 0.400 & 0.400 & 0.400 & 0.400 & 0.400 \\
\hline $\mathrm{CO}>\mathrm{EU}($ AvgPath) & 0.343 & 0.270 & 0.267 & 0.205 & 0.267 & 0.230 \\
\hline CO>EU(SEPath) & 0.067 & 0.145 & 0.151 & 0.205 & 0.157 & 0.188 \\
\hline $\mathrm{CO}>\mathrm{AC}($ TruePath) & 0.250 & 0.250 & 0.250 & 0.250 & 0.250 & 0.250 \\
\hline $\mathrm{CO}>\mathrm{AC}($ AvgPath $)$ & 0.219 & 0.174 & 0.171 & 0.129 & 0.175 & 0.151 \\
\hline CO>AC(SEPath) & 0.052 & 0.098 & 0.104 & 0.135 & 0.109 & 0.125 \\
\hline GT>SU(TruePath) & 0.500 & 0.500 & 0.500 & 0.500 & 0.500 & 0.500 \\
\hline GT>SU(AvgPath) & 0.380 & 0.321 & 0.315 & 0.260 & 0.312 & 0.280 \\
\hline GT>SU(SEPath) & 0.124 & 0.187 & 0.194 & 0.246 & 0.200 & 0.230 \\
\hline EU>SU(TruePath) & 0.230 & 0.230 & 0.230 & 0.230 & 0.230 & 0.230 \\
\hline EU>SU(AvgPath) & 0.189 & 0.181 & 0.178 & 0.152 & 0.194 & 0.177 \\
\hline EU>SU(SEPath) & 0.055 & 0.078 & 0.082 & 0.097 & 0.084 & 0.090 \\
\hline AC>SU(TruePath) & 0.200 & 0.200 & 0.200 & 0.200 & 0.200 & 0.200 \\
\hline$A C>S U(A v g P a t h)$ & 0.164 & 0.161 & 0.157 & 0.134 & 0.178 & 0.163 \\
\hline AC>SU(SEPath) & 0.054 & 0.072 & 0.076 & 0.088 & 0.078 & 0.082 \\
\hline GT3<GT(TrueLoad) & 0.700 & 0.700 & 0.700 & 0.700 & 0.700 & 0.700 \\
\hline GT3<GT(AvgLoad) & 0.811 & 0.501 & 0.468 & 0.486 & 0.433 & 0.455 \\
\hline GT3<GT(SELoad) & 0.113 & 0.213 & 0.245 & 0.237 & 0.281 & 0.267 \\
\hline
\end{tabular}

Table A3c. Monte Carlo experiment results with a sample size of 500 and $50 \%$ missing data.

\begin{tabular}{|c|c|c|c|c|c|c|}
\hline Missing data imputation scheme & NMD & MEAN & MREGR & HREGR & MSREG & HSREG \\
\hline $\mathrm{CO}>\mathrm{GT}$ (TruePath) & 0.450 & 0.450 & 0.450 & 0.450 & 0.450 & 0.450 \\
\hline CO>GT(AvgPath) & 0.389 & 0.245 & 0.250 & 0.171 & 0.238 & 0.193 \\
\hline CO>GT(SEPath) & 0.070 & 0.218 & 0.218 & 0.288 & 0.236 & 0.274 \\
\hline $\mathrm{CO}>\mathrm{EU}($ TruePath) & 0.400 & 0.400 & 0.400 & 0.400 & 0.400 & 0.400 \\
\hline $\mathrm{CO}>\mathrm{EU}($ AvgPath) & 0.343 & 0.213 & 0.216 & 0.150 & 0.209 & 0.168 \\
\hline CO>EU(SEPath) & 0.067 & 0.205 & 0.206 & 0.260 & 0.218 & 0.251 \\
\hline $\mathrm{CO}>\mathrm{AC}($ TruePath $)$ & 0.250 & 0.250 & 0.250 & 0.250 & 0.250 & 0.250 \\
\hline $\mathrm{CO}>\mathrm{AC}$ (AvgPath) & 0.219 & 0.143 & 0.144 & 0.098 & 0.140 & 0.113 \\
\hline $\mathrm{CO}>\mathrm{AC}$ (SEPath) & 0.052 & 0.133 & 0.137 & 0.168 & 0.154 & 0.168 \\
\hline GT>SU(TruePath) & 0.500 & 0.500 & 0.500 & 0.500 & 0.500 & 0.500 \\
\hline GT>SU(AvgPath) & 0.380 & 0.270 & 0.270 & 0.206 & 0.263 & 0.227 \\
\hline GT>SU(SEPath) & 0.124 & 0.240 & 0.243 & 0.301 & 0.254 & 0.285 \\
\hline EU>SU(TruePath) & 0.230 & 0.230 & 0.230 & 0.230 & 0.230 & 0.230 \\
\hline EU>SU(AvgPath) & 0.189 & 0.172 & 0.170 & 0.134 & 0.183 & 0.158 \\
\hline EU>SU(SEPath) & 0.055 & 0.098 & 0.103 & 0.119 & 0.105 & 0.115 \\
\hline AC>SU(TruePath) & 0.200 & 0.200 & 0.200 & 0.200 & 0.200 & 0.200 \\
\hline $\mathrm{AC}>\mathrm{SU}(\mathrm{AvgPath})$ & 0.164 & 0.157 & 0.158 & 0.127 & 0.175 & 0.151 \\
\hline AC>SU(SEPath) & 0.054 & 0.090 & 0.095 & 0.103 & 0.104 & 0.109 \\
\hline GT3<GT(TrueLoad) & 0.700 & 0.700 & 0.700 & 0.700 & 0.700 & 0.700 \\
\hline GT3<GT(AvgLoad) & 0.811 & 0.339 & 0.307 & 0.322 & 0.267 & 0.285 \\
\hline GT3<GT(SELoad) & 0.113 & 0.373 & 0.403 & 0.395 & 0.443 & 0.431 \\
\hline
\end{tabular}




\section{NED KOCK}

\section{Appendix B: Business Process Modeling Approaches Used}

The figure below illustrates the two types of representations used in the business process redesign projects. In the context of our data analyses example, the one on the left was coded as 1 , and the one on the right as 0 . They correspond to high and low communication flow orientations, respectively, of the business process modeling approach used.
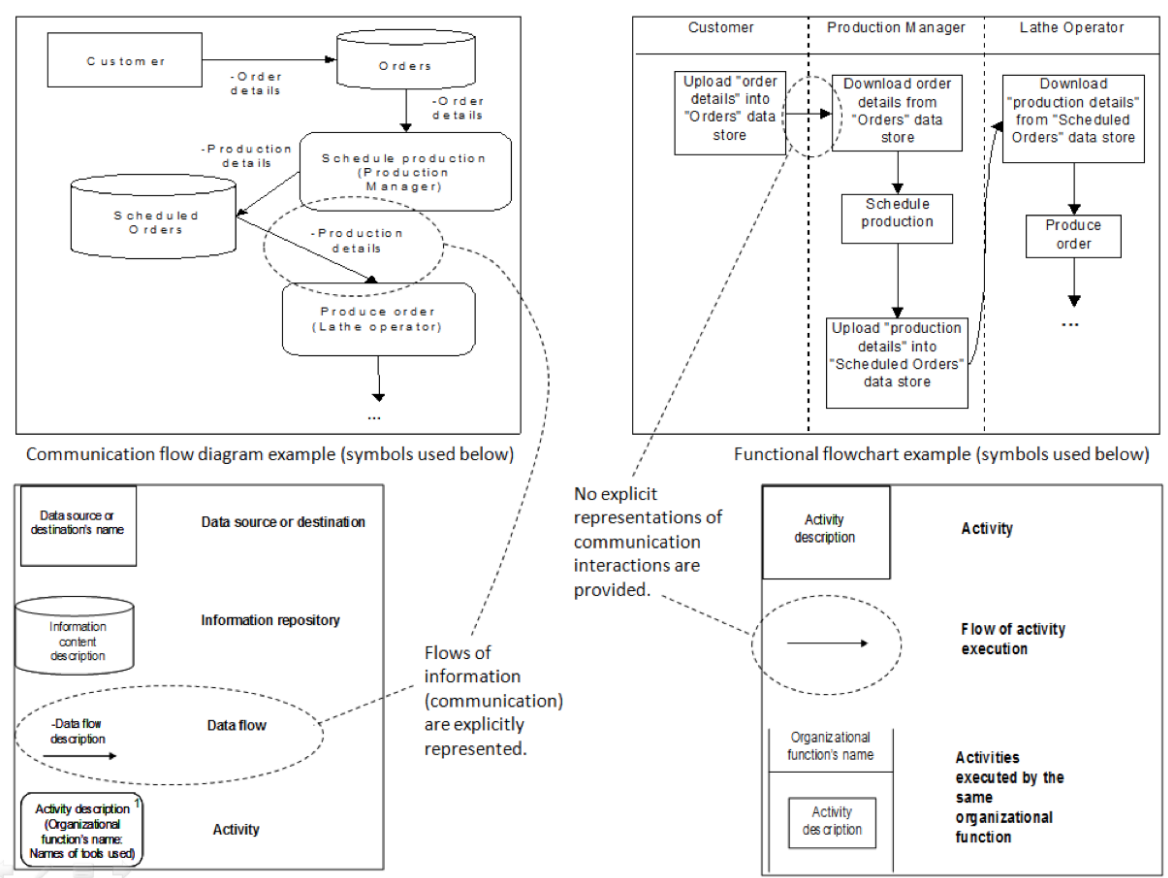

Figure A1. High (left) and low (right) communication flow orientations of the business process modeling approach. 


\section{Appendix C: Questionnaire Used in Empirical Study}

The question-statements below were used for latent variable measurement in the illustrative study. Except for communication flow orientation $\left(C_{1}\right)$, all questionstatements were answered on 7-point Likert-type scales.

\section{Communication flow orientation $\left(C_{1}\right)$}

- $\quad C_{11}$ : Coded as either 1 or 0 , corresponding to high or low communication flow orientation of the business process modeling approach used.

\section{Usefulness in the development of IT solutions $\left(C_{2}\right)$}

- $\quad C_{21}$ : This process modeling approach is useful in the development of a generic IT solution to automate the redesigned process.

- $\quad C_{22}$ : Creating a generic IT solution to enable the redesigned process is easy based on this process modeling approach.

- $\quad C_{23}$ : Graphical process representations using this approach facilitate the generation of a generic IT solution to automate the redesigned process.

\section{Ease of understanding $\left(C_{3}\right)$}

- $\quad C_{31}$ : Processes modeled using this approach are easy to understand.

- $\quad C_{32}$ : Graphical representations of processes using this approach are clear.

- $\quad C_{33}$ : This process modeling approach leads to graphical models that are easy to understand.

\section{Accuracy $\left(C_{4}\right)$}

- $\quad C_{41}$ : This process modeling approach leads to accurate process representations.

- $\quad C_{42}$ : Models created using this approach are correct representations of a process.

- $\quad C_{43}$ : Graphical representations using this approach clearly reflect the real process. 


\section{NED KOCK}

\section{Impact on redesign success $\left(C_{5}\right)$}

- $\quad C_{51}$ : Using this process modeling approach is likely to contribute to the success of a process redesign project.

- $\quad C_{52}$ : Success chances are improved if this process modeling approach is used.

- $\quad C_{53}$ : Using the graphical process representations in this approach is likely to make process redesign projects more successful. 